\title{
VISIBLE AND INFRARED RARE-EARTH ACTIVATED ELECTROLUMINESCENCE FROM ERBIUM DOPED GaN
}

\author{
M. Garter*, R. Birkhahn*, A. J. Steckl ${ }^{*}$ and J. Scofield** \\ *Nanoelectronics Laboratory, University of Cincinnati \\ Cincinnati, Ohio 45221-0030 USA, a.steck1@uc.edu \\ **Air Force Research Laboratory, Wright-Patterson AFB, OH 45433
}

\section{Cite this article as: MRS Internet J. Nitride Semicond. Res. 4S1, G11.3 (1999)}

\begin{abstract}
Room temperature visible and IR light electroluminescence (EL) has been obtained from Er-doped GaN Schottky barrier diodes. The GaN was grown by molecular beam epitaxy on $\mathrm{Si}$ substrates using solid sources (for $\mathrm{Ga}$ and $\mathrm{Er}$ ) and a plasma source for $\mathrm{N}_{2}$. Transparent contacts utilizing indium tin oxide were employed. Strong green light emission was observed under reverse bias due to electron impact excitation of the Er atoms. Weaker emission was present under forward bias. The emission spectrum consists of two narrow green lines at 537 and $558 \mathrm{~nm}$ and minor peaks at 413, 461, 665, and $706 \mathrm{~nm}$. There is also emission at $1000 \mathrm{~nm}$ and $1540 \mathrm{~nm}$ in the IR. The green emission lines have been identified as Er transitions from the ${ }^{2} \mathrm{H}_{11 / 2}$ and ${ }^{4} \mathrm{~S}_{3 / 2}$ levels to the ${ }^{4} \mathrm{I}_{15 / 2}$ ground state. The IR emission lines have been identified as transitions from the ${ }^{4} \mathrm{I}_{11 / 2}$ and ${ }^{4} \mathrm{I}_{13 / 2}$ levels to the ${ }^{4} \mathrm{I}_{15 / 2}$ ground state. EL intensity for visible and IR light has a sub-unity power law dependence on bias current. An external quantum efficiency of $0.1 \%$ has also been demonstrated under a reverse bias current of $3.85 \mathrm{~mA}$.
\end{abstract}

\section{INTRODUCTION}

The recent groundbreaking work ${ }^{1}$ by Nakamura in GaN based LEDs and laser diodes has resulted in shorter wavelength semiconductor visible light emission. At the same time novel work is being conducted using rare earth elements as sources of light emission. Results based on glasses $^{2,3}$ are exciting for display, memory and discrete device technologies. Rare-earth (RE) doped Silicon based devices ${ }^{4,5,6,7,8,9}$ have improved so that room temperature emission is now possible. III-V semiconductors doped with rare-earth elements have also been used $^{10,11,12,13,14,15,16,17,18}$ and have advantages compared to narrow bandgap materials ${ }^{19}$. The advantage of Nakamura's devices is their extremely high quantum efficiency ${ }^{1}(\sim 5 \%)$, whereas RE doped devices offer multiple color emission, wavelength-limited only by the RE element(s) chosen and not by the band gap energy of the semiconductor. Our recent work now indicates the 
possibility of combining the advantages of these two very different approaches in order to realize highly efficient full color displays in a single semiconductor host or very simple discrete devices using RE-doped GaN. We have recently reported strong visible (green) RE-activated PL from insitu Er-doped GaN grown by MBE on sapphire ${ }^{20}$ and on $\mathrm{Si}^{21}$ substrates. The same strong green RE-activated emission was also observed ${ }^{22}$ in EL from simple Al Schottky diodes on GaN:Er and from more sophisticated devices using indium tin oxide(ITO) transparent contacts. In this paper we report on the performance characteristics of Er-doped GaN LEDs emitting in both the visible and IR regions.

\section{EXPERIMENT}

The GaN:Er light emitting devices can be operated in two different ways. One way is to use both top side contacts, which consist of patterned indium tin oxide (ITO), for both positive and negative electrodes. Under reverse bias, the smaller, emitting electrode is negative and the larger electrode is positive. The devices can also be operated by making an ohmic contact to the backside of the $\mathrm{Si}$. This method is interesting because current actually flows across the GaN/Si heterojunction. The devices behave very similarly in both modes and all data presented here were taken using top-side contacts.

The Er-doped GaN was grown using a Riber MBE-32 system on 2 inch p-Si (111) substrates and on 2 inch sapphire. Solid sources were used to supply the Ga and Er fluxes, while an STVA RF-plasma source supplied atomic nitrogen. Prior to MBE growth, the Si substrate was cleaned in acetone, methanol and DI water, followed by HF to remove all native oxide. After insertion into the MBE chamber, a thin, low temperature GaN buffer layer was first grown followed by a GaN:Er layer. The GaN layers were grown at a substrate temperature between 700- $750^{\circ} \mathrm{C}$. The ITO contacts were formed using RF-sputtering in conjunction with a lift-off process. The ITO target had a composition of $90 \% \mathrm{In}_{2} \mathrm{O}_{3}$ and $10 \% \mathrm{SnO}_{2}$. The sputtering was performed at 5 mTorr with $133 \mathrm{~W}$ of RF power, resulting in -183 volts of DC bias. Under these conditions, the deposition rate was $\sim 70 \AA / \mathrm{min}$. The ITO film was $\sim 4200 \AA$ thick and had a sheet resistance of $30 \Omega$ /square as-deposited. Subsequent annealing in a nitrogen atmosphere for 2 minutes at $450{ }^{\circ} \mathrm{C}$ reduced the sheet resistance to $\sim 5.5 \Omega /$ square with transmission of $\sim 92 \%$ at a wavelength of $537 \mathrm{~nm}$ and $\sim 3 \%$ at $1.5 \mu \mathrm{m}$. Electroluminescence data were collected using either an Acton Research spectrometer or an Ocean Optics fiber optic spectrometer. Optical power 
measurements were made using a Newport 1818-SL/CM optical power meter. All measurements reported were made at room temperature.

\section{RESULTS}

Fig. 1 shows a photograph of a GaN:Er LED in operation using a standard probe station. In this photo, negative bias is being applied to the green light emitting electrode, resulting in a current of $19 \mathrm{~mA}$. The device shown here is fairly large ( $2 \mathrm{~mm}$ radius) because it was designed to make spectral data collection using an optical fiber more practical. An optical fiber can be placed in the immediate vicinity of the device, while still leaving enough room for an electrical probe tip. The emission intensity and color is uniform across the diode and the emitted light is easily seen in a normally lit room. Fig. 2 shows the difference in spectral shape between a commercial GaPbased device and our GaN:Er device. Notice that the conventional GaP LED is shifted more toward the yellow end and has an FWHM of $30 \mathrm{~nm}$. By comparison, the GaN:Er green device has an FWHM of 5-6 nm and emits in the middle of the green range. The GaP LED was biased at $2 \mathrm{~mA}$ and the GaN:Er device was biased at $10 \mathrm{~mA}$ during these measurements. The peaks represent the actual signal intensity. Minor peaks at $413 \mathrm{~nm}$ and $665 \mathrm{~nm}$ are also present in the GaN:Er device spectrum. Fig. 3 shows the IR emission spectrum from a GaN:Er device biased at 75 volts and $19 \mathrm{~mA}$. We have identified the $1.5 \mu \mathrm{m}$ radiation preiously. ${ }^{23}$ The $1 \mu \mathrm{m}$ peak corresponds to a transition from ${ }^{4} \mathrm{I}_{11 / 2}$ to the ${ }^{4} \mathrm{I}_{15 / 2}$ ground state. It has an FWHM of $\sim 25 \mathrm{~nm}$. The

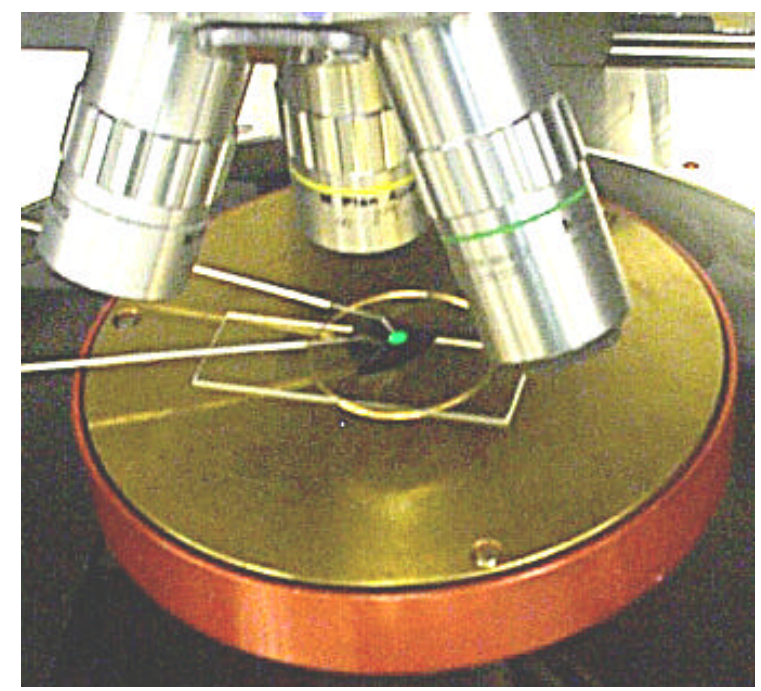

Fig. 1 Photograph of visible rare-earthactivated emission from ITO/GaN:Er device.

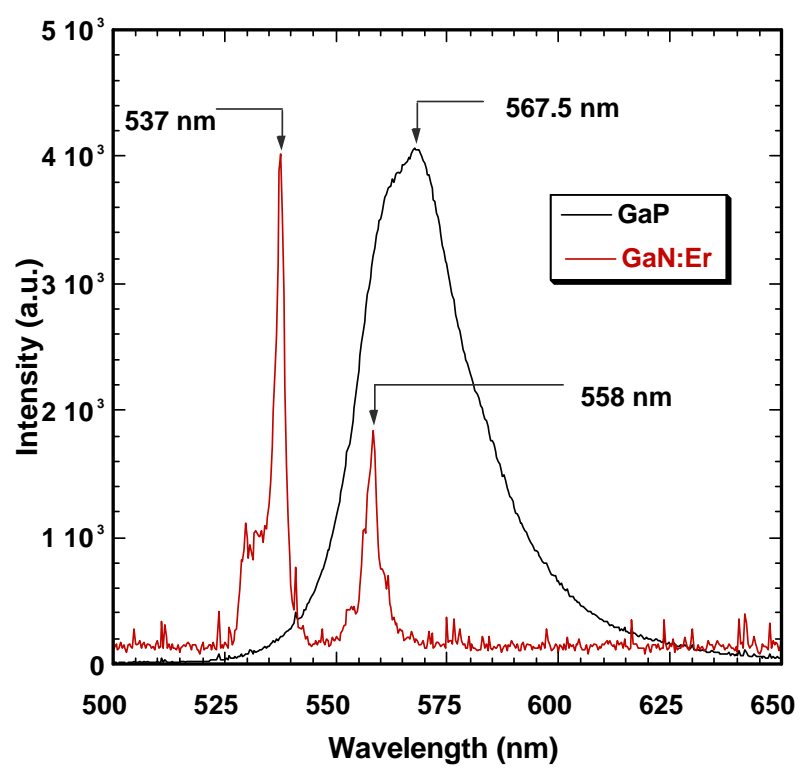

Fig. 2 Emission spectrum in the visible range from a commercial green GaP-based LED vs. our GaN:Er device. 
visible and IR optical power emitted and detected from a GaN:Er LED are plotted as a function of bias current in Fig. 4. The visible optical power at the detector has a sub-unity power law dependence on bias current over the measurement range, reaching a value of $212 \mathrm{nW}$ at $19 \mathrm{~mA}$. The IR optical power also increases with a power law dependence over the measurement range reaching a value of $12.3 \mathrm{nW}$ at $19 \mathrm{~mA}$. It is important to note that the ITO electrodes for these experiments were optimized for green light, but the transmission in the IR can be increased by processing the ITO differently. The ITO layer transmits about 3\% of the IR light emitted and about $92 \%$ of the visible green light. With this in mind, our devices probably emit slightly more IR light than visible light, however, the ITO absorbs most of the IR light. During these measurements, a Si photodetector was placed $2.0 \mathrm{~cm}$ above the device. Assuming a point source of radiation, and uniform hemispherical emission, the visible optical power generated at the device is estimated to be $5.33 \mu \mathrm{W}$ for $10 \mathrm{~mA}$ of applied reverse bias. Under these conditions, the external quantum efficiency at $537 \mathrm{~nm}$ is estimated to be $0.012 \%$. However, a maximum external quantum efficiency of $0.1 \%$ has been reached for a GaN:Er device grown on sapphire. This device was operated at 155 Volts and $3.85 \mathrm{~mA}$, and it was also used to take the data presented in Fig. 5. Fig. 5 summarizes the EL peaks that we have observed to date. The number of observable peaks increases with increasing applied power. The following peaks have been observed during EL of GaN:Er: 413, 461, 537, 558, 665, 671, 706, 1000, 1515, 1539, 1555nm. The EL peaks at 537 and $558 \mathrm{~nm}$ are dominant in the visible range and correspond to transitions from the ${ }^{2} \mathrm{H}_{11 / 2}$ and the ${ }^{4} S_{3 / 2}$ to the ${ }^{4} I_{15 / 2}$ ground state. It is interesting to note that the $706 \mathrm{~nm}$ line probably

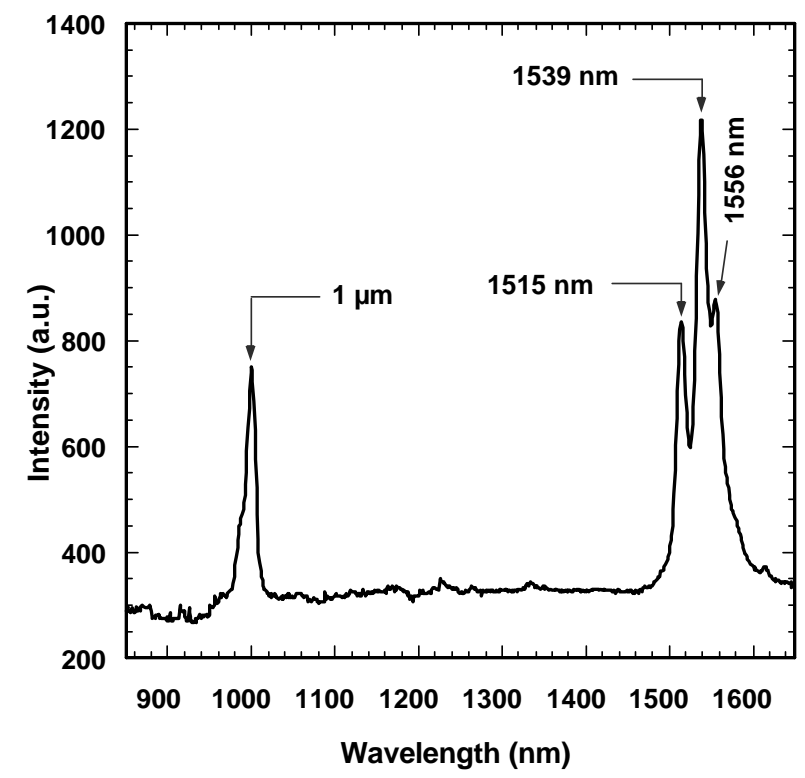

Fig. 3 Rare-earth activated emission spectra in the IR range from an ITO/GaN:Er device.

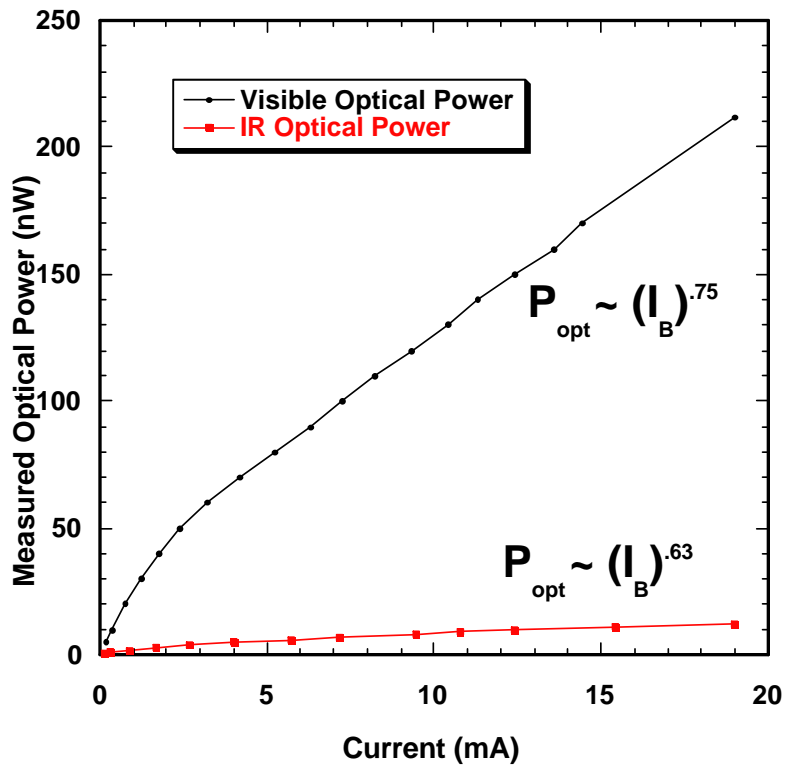

Fig. 4 Visible and IR optical power as a function of bias current. 


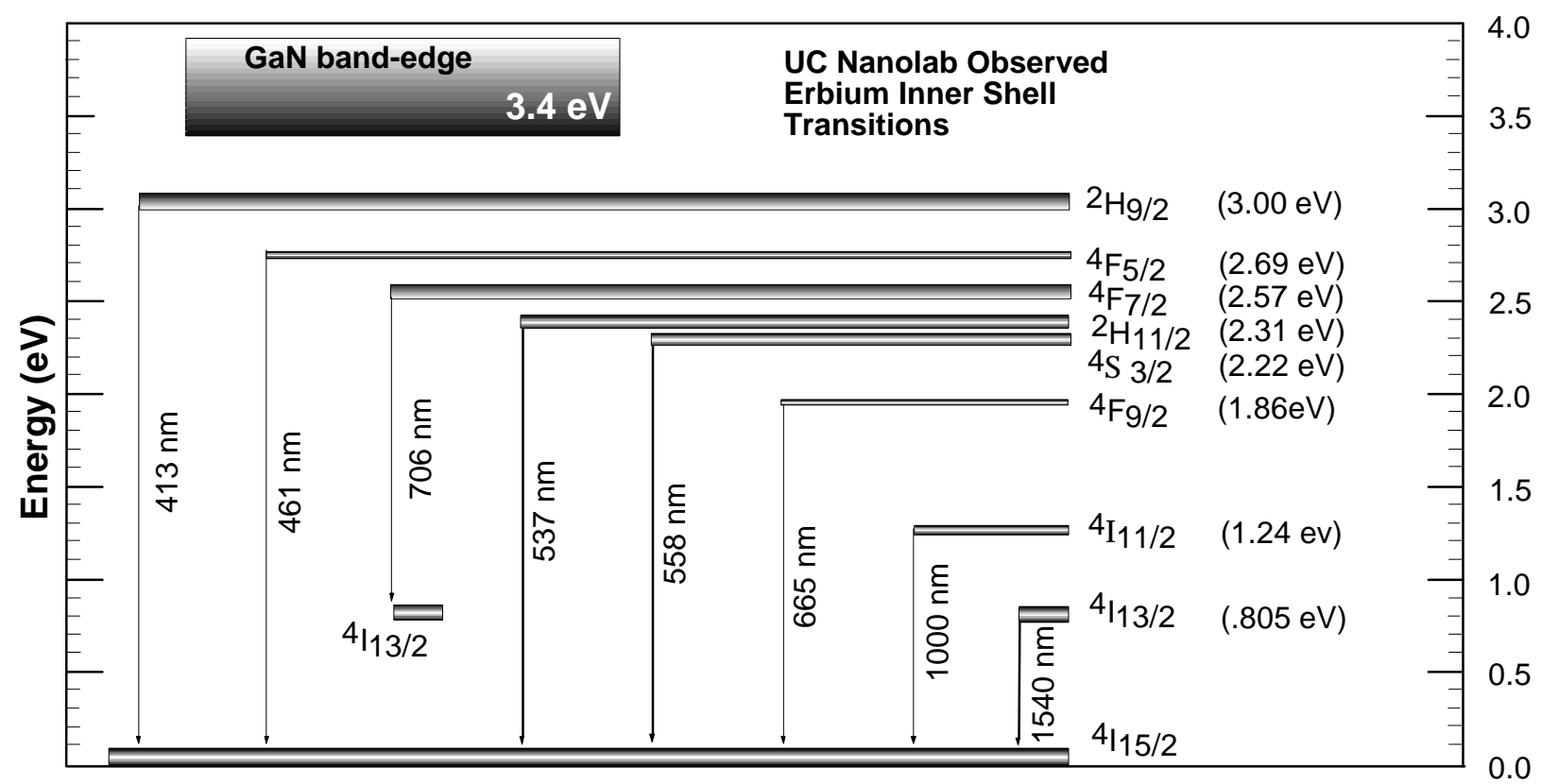

Fig. 5 Summary of the observed atomic energy levels of Rare-earth-activated emission in the visible and IR range from an ITO/GaN:Er device.

corresponds to a transition from the ${ }^{4} \mathrm{~F}_{7 / 2}$ to the ${ }^{4} \mathrm{I}_{13 / 2}$ state instead of the normal ground state. This is the only intermediate transition we have observed. In addition, it is interesting that the ${ }^{4} \mathrm{I}_{9 / 2}$ $(\sim 1.55 \mathrm{ev}$ above the ground state) level, a common transition in other host materials, is not observed in our material.

\section{SUMMARY}

In summary, we have reported visible and IR rare-earth-activated electroluminescence of Er-doped GaN. The use of ITO as a contact provides a fast and easy way to make devices, and also increases the amount of visible light output from the device. Both IR and visible EL peaks show narrow linewidths. Our devices transmit appreciably more visible than IR light, however the use of ITO as a contact material is not ideal for IR light emission under the specific conditions that the ITO was deposited. Future work will focus on reducing the operating voltage and increasing the external quantum efficiency of the devices. This work was supported in part by a BMDO/ARO contract (L. Lome and J. Zavada) and AASERT grants. The authors would like to acknowledge the support and encouragement of J. Zavada. Equipment support was provided by an ARO URI grant and the Ohio Materials Network. 


\section{REFERENCES}

${ }^{1}$ S. Nakamura and G. Fasol, The Blue Laser Diode, Springer Verlag, Berlin (1997), p. 215.

${ }^{2}$ E. Downing, L. Hesselink, J. Ralston and R. Macfarlane, Science 273, 1185 (August 1996).

${ }^{3}$ A.C. Tropper, J.N. Carter, R.D.T. Lauder, D.C. Hanna, S.T. Davey, and D. Szebesta, J. Opt. Soc. Am. B 11 (5), 886 (May 1994).

${ }^{4}$ H. Ennen, G. Pomrenke, A. Axmann, K. Eisele, W. Haydl, and J. Schneider, Appl. Phys. Lett. 46 (4), 381 (February 1985).

${ }^{5}$ G. Franzo and F. Priolo, Appl. Phys. Lett. 64, 2235 (April 1994).

${ }^{6}$ B. Zheng, J. Michel, F. Y. G. Ren, L. C. Kimerling, D. C. Jacobson, and J. M. Poate, Appl. Phys. Lett. 64, 2842 (May 1994).

${ }^{7}$ S. Lombardo, S. U. Campisano, G.N. van den Hoven, and A. Polman, J. Appl. Phys. 77, 6504 (June 1995)

${ }^{8}$ C. X. Du, W. X. Ni, K. B. Joelsson, and G. V. Hansson, Appl. Phys. Lett. 71, 1023 (August 1997).

${ }^{9}$ S. Coffa, G. Franzo, and F. Priolo, MRS Bulletin, 25 (April 1998).

${ }^{10}$ R. G. Wilson and R. N. Schwartz, C. R. Abernathy and S. J. Pearton, N. Newman, M. Rubin, and T. Fu, J. M. Zavada, Appl. Phys. Lett. 65 (8), 992 (August 1994).

${ }^{11}$ S. Kim, S. J. Rhee, D. A. Turnbull, E. E. Reuter, X. Li, J. J. Coleman, and S. G. Bishop, Appl. Phys. Lett. 71 (2), 231 (July 1997).

${ }^{12}$ S. Kim, S. J. Rhee, D. A. Turnbull, X. Li, J. J. Coleman, and S. G. Bishop, Appl. Phys. Lett. 71 (18), 2662 (November 1997).

${ }^{13}$ D. M. Hansen, R. Zhang, N. R. Perkins, S. Safvi, L. Zhang, K. L. Bray, and T. F. Kuech, Appl. Phys. Lett. 72 (10), 1244 (March 1998).

${ }^{14}$ E. Silkowski, G. S. Pomrenke, Y. K. Yeo and R. L. Hengehold, Physica Scripta T69, 276 (1997).

${ }^{15}$ J. T. Torvik, C. H. Qiu, R. J. Feuerstein and J. I. Pankove, F. Namavar, J. Appl. Phys. 81 (9), 6343 (May 1997).

${ }^{16}$ C. H. Qiu, M. W. Leksono, and J. I. Pankove, J. T. Torvik and R. J. Feuerstein, F. Namavar, Appl. Phys. Lett. 66 (5), 562 (January 1995).

${ }^{17}$ J. M. Zavada and D. Zhang, Solid-State Electron. 38, 1285 (1995).

${ }^{18}$ J. T. Torvik, R. J. Feuerstein, J. I. Pankove, C. H. Qiu, and, F. Namavar, Appl. Phys. Lett. 69, 2098 (September 1996).

${ }^{19}$ J. M. Zavada, M. Thaik, U. Hommerich, J.D. Mackenzie, C. R. Abernathy, Electrochem. Soc. 98-1, $193^{\text {rd }}$ Meeting, Abstract no. 535, (Spring 1998).

${ }^{20}$ A. J. Steckl and R. Birkhahn, Appl. Phys. Lett. 73 (12), 1700 (Sept. 21, 1998).

${ }^{21}$ R. Birkhahn and A. J. Steckl, Appl. Phys. Lett. 73 (14), 2143 (Oct. 12, 1998).

${ }^{22}$ A. J. Steckl, M. Garter, R. Birkhahn, J. Scofield, Appl. Phys. Lett. 73 (17), 2450 (Oct. 26, 1998).

${ }^{23}$ M. Garter, J. Scofield, R. Birkhahn, A. J. Steckl, Appl. Phys. Lett. 74 (2), 182 (Jan. 11, 1999). 